\title{
Editorial
}

\section{Preserving and Exploiting Molecular Diversity: Deposit and Exchange of Chemical Information and Chemical Samples}

\author{
Shu-Kun Lin \\ Molecular Diversity Preservation International (MDPI), Saengergasse 25, CH-4054 Basel, Switzerland. Tel. +41 79322 \\ 3379; Fax +41613028918 (Lin@mdpi.org)
}

Received: 27 December 1996 / Accepted: 7 January 1997 / Published: 17 January 1997

Starting with volume 2 of the journal Molecules [1], the nonprofit international organization MDPI will provide, in addition to the sample deposit services, the service for the deposit of information (experimental data, particularly spectroscopic data). Preserving and exploiting molecular diversity for both information and samples will continue to be the goal the journal Molecules and the organization MDPI strives for.

Unlike other fields of science, chemists contribute not only a large volume of knowledge (chemical information) but also virtual substance (chemical samples) of various molecules. These two aspects of contribution constitute the precious molecular diversity [2].

However, we know that most of the chemical samples exist only on paper. Nevertheless, the loss of chemical samples [3] can be avoided by coordinated efforts of chemical samples registration, collection, deposit and exchange, in addition to the peer-reviewed high-quality publication of normal research reports. Molecules is the first chemistry journal to encourage authors to register and exchange their available chemical samples. This has been quite successful for the publication of the first volume in 1996 and has set an example to other chemistry journals [4]. The editor will continue to insist on this policy.

We intend to publish in volume 2 very short posters of synthetic works and the data of the structural characteriza- tion which can be one molecule (one structure) per paper and can be as short as one page only. Such publications in Molecules will serve as the experimental data deposit. This project is interesting because a large volume of very precious chemical information, particularly the very diverse works of synthesis and structural characterization have either never been published or the submitted papers were rejected by editors because they were too trivial (normally because it belonged to the classical scope of chemistry, i.e., pure synthetic or pure spectroscopic measurements), or the chemists themselves never planned to publish such works for individual compounds and isolated data because they thought them not publishable in a traditional journal. We believe that synthesis and structural elucidation of individual compounds are still the essence of chemistry and the material foundation of other research.

The ready publication of all scattered unassembled data for individual compounds in Molecules as short posters will provide a bank for chemists to deposit all of their information of synthesis and structural characterization, together with the sample availability information. Those works that have no compound samples available will also be published. When a large volume of (say 1 million) structures is published in this way and also constructed as a retrievable databank, it will be a very useful treasure to all chemists and other related scientists. If every synthetic chemist contributes 100 
such posters, this number will be easily reached within several years.

\section{References and Notes}

1. Starting with volume 2, 1997, Molecules (ISSN 14203049) will be published by Molecular Diversity Preservation International (MDPI). Volume 1, 1996 was published in collaboration with Springer-Verlag.

2. Lin, S. -K. Molecular diversity assessment: Logarithmic relations of information and species diversity and logarithmic relations of entropy and indistinguishability after rejection of Gibbs paradox of entropy of mixing. Molecules 1996, 1, 57-67.

3. The stability of samples and the purity of old compound samples have been the main concern of many chem- ists. We found that in many pharmaceutical companies, all the solid samples prepared more than 50 years ago and stored even at room temperature have so far been found to be applicable and can be used for such purposes as high throughput screening. Many of the MDPI compounds can be used for high throughput screening where several compounds are mixed by the users before the bioactivity tests. The possible impurities in a sample are not a problem for such tests. According to our investigations, many chemical reagent suppliers store most of their chemicals at $\mathrm{rt}$ for several years. The decay of compounds is greatly accelerated only if they are exposed to air, light, or are not in solid form.

4. Several chemistry journals have agreed to encourage samples deposit and exchange. For details, visit the www.mdpi.org/forum.htm homepage. 